\title{
GMR
}

\section{miR-577 inhibits pancreatic $\beta$-cell function and survival by targeting fibroblast growth factor 21 (FGF-21) in pediatric diabetes}

\author{
X.Y. Chen ${ }^{2}$, G.M. Li' ${ }^{1}$, Q. Dong ${ }^{1,2}$ and H. Peng ${ }^{1,3}$ \\ ${ }^{1}$ Pediatric Department, Shandong Provincial Hospital Affiliated to Shandong University, \\ Jinan, China \\ 2Pediatric Department, Taian City Central Hospital, Tai'an, China \\ ${ }^{3}$ Pediatric Department, Jinan Central Hospital affiliated to Shandong University, \\ Jinan, China \\ Corresponding author: G.M. Li \\ E-mail: liguimei20150423@163.com \\ Genet. Mol. Res. 14 (4): 15462-15470 (2015) \\ Received April 23, 2015 \\ Accepted July 22, 2015 \\ Published November 30, 2015 \\ DOI http://dx.doi.org/10.4238/2015.November.30.24
}

\begin{abstract}
Pancreatic $\beta$-cell dysfunction is a central component of the pathogenesis of pediatric diabetes. MicroRNA (miRNA) have become one of the most encouraging and fruitful fields in biological research, and have been implicated as new players in the pathogenesis of diabetes and diabetes-associated complications. The role of miRNA in diabetes begins with the development of pancreatic islets. Fibroblast growth factor (FGF)21 enhances glucose uptake in adipocytes, protecting transgenic animals from diet-induced obesity when overexpressed, and lowers blood glucose and triglyceride levels in diabetic animals (when administered); therefore, it is a good way to treat diabetes. However, the mechanism of miRNA in regulation of FGF21 is not known. In this study, FGF-21 was predicted to be the target of miR-577. Therefore, we investigated the effects of miR-577 on $\beta$-cell function and survival by targeting FGF-21. We demonstrated that, although FGF-21 does not acutely stimulate insulin secretion in isolated islets from normal rats, it increases insulin secretion and insulin content
\end{abstract}


in diabetic islets and protects $\beta$-cells from apoptosis via the activation of extracellular signal-regulated kinase $1 / 2$ and Akt signaling pathways.

Key words: miR-577; FGF-21; Pancreatic $\beta$-Cell

\section{INTRODUCTION}

Pancreatic $\beta$-cell dysfunction is a central component of the pathogenesis of pediatric diabetes. Type 1 diabetes manifests as a result of the autoimmune destruction of $\beta$-cells, whereas type 2 diabetes is characterized by reduced $\beta$-cell mass and marked functional defects, including impaired first-phase insulin secretion, increased proinsulin-to-insulin ratio, and an elevated rate of $\beta$-cell apoptosis. The amount of secreted insulin is determined by the secretory activity of the $\beta$-cell and the total number of $\beta$-cells in the pancreas. Glucose plays an essential role in the control of $\beta$-cell secretory activity. Glucose metabolism leads to an increase in the ATP/ADP ratio, membrane depolarization, $\mathrm{Ca}^{2+}$ influx, and stimulated insulin secretion (Zheng et al., 2012; Schaap et al., 2013; Wang et al., 2013).

Members of the fibroblast growth factor (FGF) family play important roles in defining and regulating the development and function of endocrine tissues; in addition, these factors modulate various metabolic processes (Cong et al., 2013; Huang et al., 2013; Kharitonenkov and Adams, 2013). FGFs modulate cellular activity via five distinct high-affinity FGF receptors (Eswarakumar et al., 2005). FGF-21 is a recently described member of the FGF family (Nishimura et al., 2000). We have previously demonstrated that FGF-21 is a potent regulator of glucose homeostasis (Kharitonenkov et al., 2005). FGF-21 enhances glucose uptake in adipocytes; FGF21 overexpression protects transgenic animals from diet-induced obesity, and its administration to diabetic animals lowers their blood glucose and triglyceride levels.

New approaches must be employed to investigate diabetes in order to obtain a deeper understanding of its pathogenesis, and for the development of novel therapeutic strategies. The use of microRNA (miRNA) has recently emerged as an encouraging and fruitful field in biological research; miRNAs have also been implicated as new players in the pathogenesis of diabetes and diabetes-associated complications. The role of miRNA in diabetes starts as early as the development of pancreatic islets. miR-124a2 and miR-375 are involved in pancreatic beta-cell development (Baroukh et al., 2007; Keller et al., 2012), and are required for the proper formation of pancreatic islets in vertebrates. miR- 375 also plays a role in the development of $\beta$-cells in mice (Keller et al., 2012), establishment of normal pancreatic endocrine cell mass during the postnatal period, and maintenance of cell viability (Poy et al., 2009).

In this study, we have discovered a significant increase in miR-577 expression in blood samples obtained from diabetic children; FGF-21 was predicted to be the target of miR-577. Subsequently, we investigated the effects of miR-577 on $\beta$-cell function and survival by targeting FGF-21.

\section{MATERIAL AND METHODS}

\section{Embryonic pancreatic $\beta$-cell (EPB) isolation}

EPB were isolated according to a previously described protocol. The INS-1 cell line, a well differentiated mouse insulinoma $\beta$-cell line that displays the characteristics of pancreatic beta-cells (including insulin secretion) in response to glucose stimulation, was cultured in Dulbecco's modified 
Eagle's medium containing high glucose (Sigma-Aldrich, St. Louis, MO, USA), supplemented with $10 \%$ fetal bovine serum and $70 \mu \mathrm{M} \beta$-mercaptoethanol, at $37^{\circ} \mathrm{C}$ in a humidified incubator with $95 \%$ air and $5 \% \mathrm{CO}_{2}$.

\section{Insulin secretion assay}

Insulin secretion in pancreatic islets was performed as described in a previous study, using three islets in $300 \mu \mathrm{L}$ Earle's balanced salt solution (Invitrogen, Carlsbad, CA, USA) supplemented with glucose and the test substances, as indicated. In order to determine the insulin content, the islets were subjected to a lysis buffer containing $75 \%$ ethanol, $23.5 \%$ water, and $1.5 \%$ concentrated $\mathrm{HCl}$. Insulin concentrations in the collected supernatant and islet lysates were measured by enzyme-linked immunosorbent assay (ELISA).

\section{RNA isolation and real-time reverse transcriptase polymerase chain reaction (RT-PCR)}

Total RNA was isolated from the cells using a commercial TRIzol reagent (Invitrogen), according to the manufacturer protocols. Briefly, the cells were lysed in TRIzol and mixed with chloroform. The lysate was centrifuged to separate RNA, DNA, and protein; total RNA was recovered, precipitated with isopropanol, washed in $75 \%$ ethanol to remove impurities, and finally dissolved in water. Subsequently, $2 \mu \mathrm{g}$ RNA was treated with DNase (in order to remove the contaminating DNA) prior to reverse transcription to CDNA using the SYBR ${ }^{\circledR}$ PCR Kit (TaKaRa Bio Inc., Tokyo, Japan). The mRNA expression was measured by performing real-time RT-PCR using a sequence detector (ABIPrism; Applied Biosystems, Foster City, CA, USA). The primers were purchased from Invitrogen. The relative expression levels were calculated by comparing the $\mathrm{Ct}$ values of the samples with those of the reference; all data was normalized to the internal control (GAPDH) values.

\section{Western blot analysis}

Western blots were performed as described elsewhere. In brief, the cells were lysed in RIPA buffer containing a $1 \mathrm{X}$ protease inhibitor cocktail; protein concentrations were determined using the Bradford assay (Bio-Rad, Hercules, CA, USA). The proteins were separated by sodium dodecyl sulfate-polyacrylamide gel electrophoresis (12.5\% SDS-PAGE) and transferred to membranes (EMD Millipore, Bedford, MA, USA) at $55 \mathrm{~V}$ for $4 \mathrm{~h}$ at $4^{\circ} \mathrm{C}$. The membranes were blocked in $5 \%$ nonfat dry milk in TBS, incubated with primary antibodies $(1: 1,000)$ in TBS overnight at $4^{\circ} \mathrm{C}$, and washed thrice with TBS-Tween 20 . The cells were then incubated with secondary antibodies conjugated with horseradish peroxidase (1:5000) in TBS, for $1 \mathrm{~h}$ at room temperature. The membranes were washed again (thrice) in TBS-Tween 20 at room temperature. Protein bands were visualized on an X-ray film, using an enhanced chemi-luminescence detection system.

\section{Immunoblotting}

INS-1E cells were lysed in radioimmunoprecipitation buffer $(50 \mathrm{mM}$ Tris- $\mathrm{HCl}, \mathrm{pH} 8.0$; $150 \mathrm{mM} \mathrm{NaCl}, 1 \% \mathrm{NP}-40,0.5 \%$ Na-deoxycholate, $5 \mathrm{mM}$ EDTA, and $0.1 \%$ SDS) containing a protease inhibitor cocktail (Roche Applied Science, Indianapolis, IN, USA). Protein samples were separated by SDS-PAGE, transferred to a polyvinylidine fluoride membrane, and probed 
with antibodies against the following proteins: Akt, phospho-Akt (Ser473), 90-kDa ribosomal S6 kinase (p90RSK), phospho-p90RSK (Ser380), phospho-ERK1/2 (Thr202/204), S6 ribosomal protein, and phospho-S6 ribosomal protein (Ser235/236; Cell Signaling Technology, Beverly, MA, USA); ERK1/2 and phospho-BAD (Ser136; Upstate, Charlottesville, VA, USA); and anti-BAD (BD Biosciences, San Diego, CA, USA). A VersaDoc imaging system (Bio-Rad) and Quantity One 1-D analysis software (version 4.4; Bio-Rad) were used to quantify the western blots.

\section{Oligonucleotide construction and lentiviral transduction}

The oligonucleotide of mature miR-577 antagomir was chemosynthesized, amplified, and cloned into GV232-Puro Vectors by Genechem Co., Ltd. (Shanghai, China). The correct sequences and insertions were confirmed by DNA sequencing. Cells were lentivirally transfected with either the GV232-Puro-miR-577 recombined vector (miR-26a) or emptyGV232-Puro vector (negative control, miR-NC). Oligonucleotide transfection or lentivirus construction was performed using Lipofectamine 2000 (Invitrogen) according to the manufacturer protocols. The transduced cells (cell density of over $40 \%$ confluence) were exposed to puromycin dihydrochloride (1 mg/ $\mathrm{mL}$; Sigma-Aldrich) for resistance selection. Following the killing of all cells in the non-transfected control culture, puromycin-resistant cell clones were picked and passaged in medium containing puromycin $(0.5 \mathrm{mg} / \mathrm{mL})$ during the first round of selection. Lentivirus-mediated silencing of miR-26a was verified by $\mathrm{qRT}$-PCR and western blot analysis.

\section{Statistical analysis}

Results are reported as means \pm standard error values for the indicated number of experiments. Statistical significance was calculated using the the Student $t$-test for data pairs, Dunnett test for multiple comparisons to a control, and Tukey test for multiple comparisons between groups.

\section{RESULTS}

\section{miR-577 is a direct target gene of FGF-21 in EPB cells}

The mechanisms by which miR-577 inhibited FGF-21-induced function were investigated. Bioinformatic analysis showed that FGF-21 was directly suppressed by miR-577 (Figure 1A). As shown in Figure 1B, the luciferase activity of pGL4-FGF-21-WT in INS-1 cells was much lower than that in control cells. The luciferase activity of pGL4-FGF-21-Mut was rescued in EPB cells. We then examined whether miR-577 could regulate endogenous FGF-21 expression in EPB and INS-1 cells. Compared to the control, endogenous FGF-21 mRNA levels (Figure 1C) were downregulated upon transfecting cells with miR-577. The FGF-21 protein levels in the cells increased under the effect of anti-miR-577 (Figure 1D).

\section{miR-577 decreases insulin content (but not insulin secretion) in EPB cells by negative regulation of FGF-21}

FGF-21 increases the insulin content in EPB cells, but not the insulin secretory capacity. We attempted to investigate the role of miR-577 in increasing the insulin content. The acute addition of $50 \mathrm{nM}$ FGF-21 to EPB cells for 4-48 $\mathrm{h}$ with FGF-21 did not affect the subsequent insulin 
secretion (induced by simulation with 3 or $15 \mathrm{mM}$ glucose; Figure 2A). However, the incubation of EPB cells for $24 \mathrm{~h}$ with $50 \mathrm{nM}$ FGF-21 elevated the insulin mRNA levels (Figure 2B), and resulted in a time-dependent increase in insulin content (Figure 2C). The increase in insulin content reached a maximum value (230\%) following incubation with FGF-21 for $8 \mathrm{~h}$; this remained relatively constant for up to $72 \mathrm{~h}$ of exposure to FGF-21 (Figure $2 \mathrm{C}$ ).

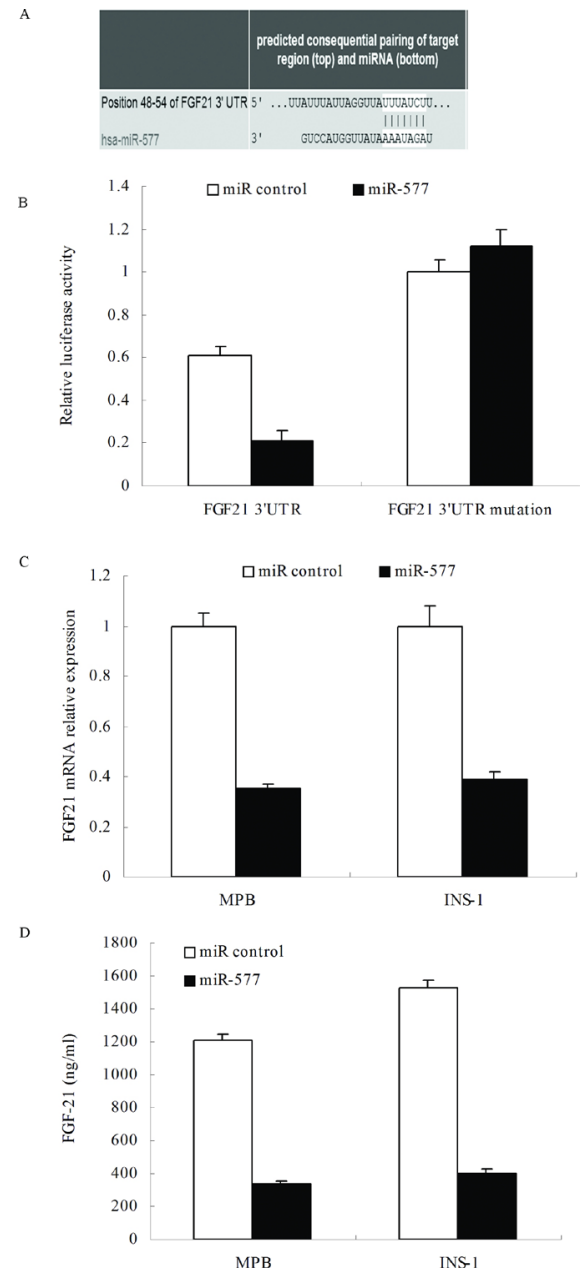

Figure 1. Restoration of miR-577 down-regulates FGF-21 expression. A. According to bioinformatic analyses, the 3'-UTR of the FGF21 gene contains binding sites for miR-577. B. miR-577 suppresses the expression of a luciferase reporter gene harboring the 3'-UTR of FGF21. The pGL4 plasmid was modified by adding the human 3'-UTR or the 3'-UTR with mutations in regions complementary to miR-577 seed regions behind the firefly luciferase gene. EPB are reported cells were transiently co-transfected with negative control (mock) or miR-577, together with the indicated luciferase constructs, and luciferase activity was analyzed after $48 \mathrm{~h}$. All data has been presented as relative firefly luciferase activity, normalized to Renilla luciferase activity from the same construct. The data are reported as means \pm standard deviation of three independent experiments. C. miR-577 restoration down-regulated FGF21 in EPB cells. Cells were transfected with miR-577 or miR control for $48 \mathrm{~h}$; subsequently, the supernatant was collected for ELISA. D. miR-577 restoration down-regulated FGF21 in EPB cells. Cells were transfected with miR-577 or miR control for 48 h, and subsequently collected for real-time PCR. 
A
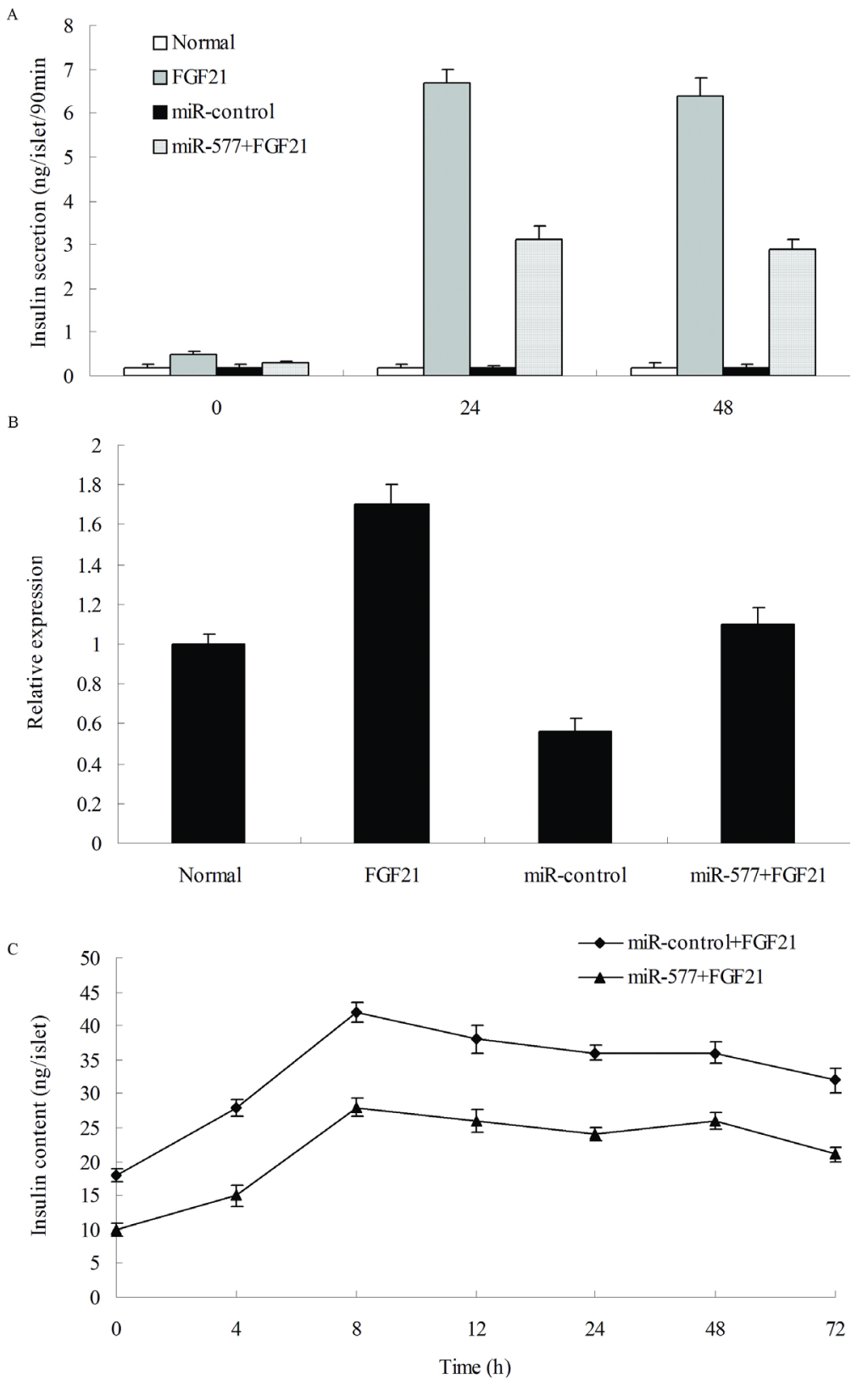

Figure 2. miR-577 decreases insulin mRNA and insulin content, but does not modulate insulin secretion. A. EPB cells infected with LV-miR-577 or LV-miR-control were treated for $0,4,8,24$, and 48 h with 50 nM FGF-21 before exposure to 3 or $15 \mathrm{mM}$ glucose for $90 \mathrm{~min}$. Results are reported as means \pm standard error (SE) of six individual experiments. B. Relative insulin gene mRNA expression in EPB cells with LV-miR-577 or LV-miR-control, under basal conditions and after exposure to FGF-21 (50 nM) for $24 \mathrm{~h}$. Results are reported as means \pm SE of four separate experiments. C. Insulin content in EPB cells with LV-miR-577 or LV-miR-control, exposed to $50 \mathrm{nmol} / \mathrm{L}$ FGF-21. Results are reported as means \pm SE of six individual experiments. 


\section{miR-577 inhibits FGF-21 activated FGF receptor downstream signaling cascades in $\beta$-cells}

The FGF-21 targeting effect of miR-577 on ERK1/2 phosphorylation was further examined in EPB cells, using an ERK1/2-specific ELISA test. The stimulatory effect of FGF-21 on ERK1/2 phosphorylation was found to be concentration-dependent (Figure 3A). Figure 3B showed that miR-577 inhibits FGF21-activated ERK1/2 signal pathway by western blot. This data suggested that miR-577 inhibits the FGF-21-induced ERK1/2 activation in EPB cells through the stimulation of a classical receptor tyrosine kinase signaling pathway.

A

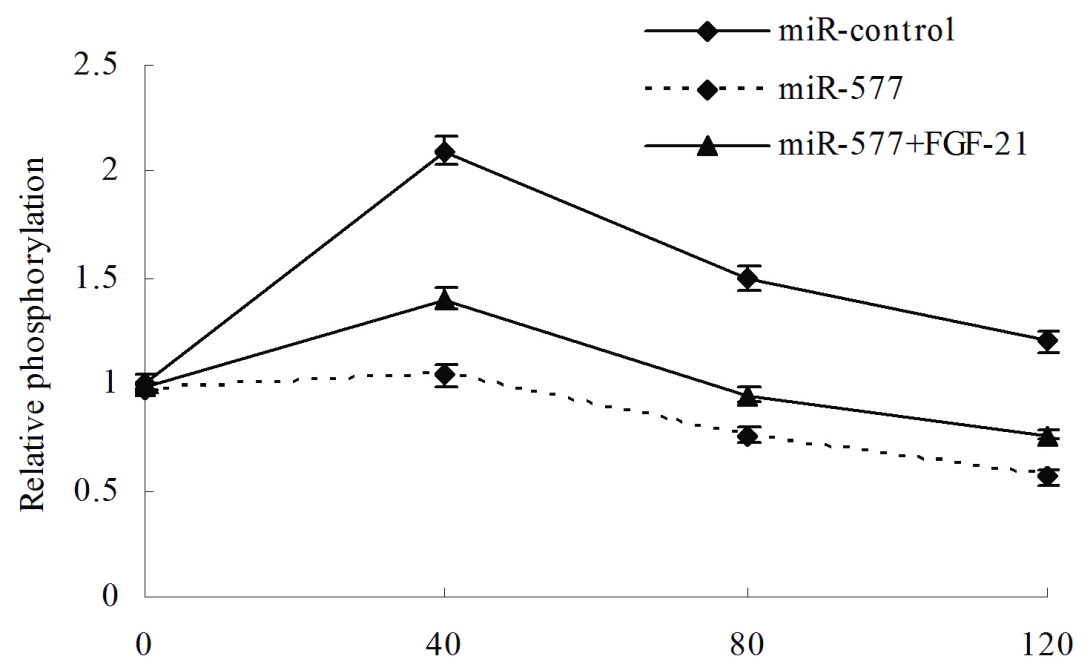

Time (min)

B

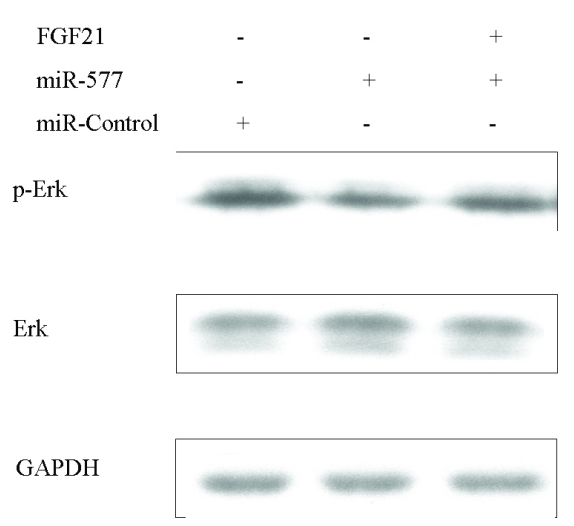

Figure 3. miR-577 inhibits FGF-21-activated FGF receptor downstream signaling cascades in $\beta$-cells. A. Doseresponse curve for stimulation of ERK1/2 activity by FGF-21 in EPB cells, as measured by ELISA. B. EPB cells were infected with lentivirus-mediated miR-577, miR-control, or a combination of miR-577 and FGF21 treatment; the proteins were isolated, and the samples were blotted for the antibodies. 


\section{DISCUSSION}

In this study, the role of miR-557 in the regulation of pancreatic islet function was investigated (by targeting) in mouse models of embryonic islets. The obtained data showed that miR-557 is a novel regulator of FGF-21, and inhibits insulin secretion and content, and inactive FGF-21 downstream signaling pathway.

FGF-21 is a typical FGF molecule that stimulates the activation of ERK1/2 and Akt signaling pathways. FGF-21 exerts a transient effect on ERK1/2 and Akt activity in cells; this activity returns to a basal level within $2 \mathrm{~h}$. Glucose-induced activation and nuclear translocation of ERK1/2 stimulates insulin gene transcription in pancreatic $\beta$-cells (Poy et al., 2009; Kim et al., 2013). However, ERK1/2 need not be activated to stimulate insulin secretion (Adams et al., 2013; Gaich et al., 2013). Our data showed that miR-577 inhibits FGF-21 activated insulin biosynthesis in pancreatic $\beta$-cells, when treated with glucose. The effect of FGF-21 on insulin content was mediated through the activation of ERK1/2, and abolished by miR-577. However, the ERK1/2 activation and increased insulin content did not translate into higher glucose-induced insulin secretion in $\beta$-cells cultured without glucose. These results supported the view that insulin biosynthesis is not ratelimiting for glucose-induced insulin secretion under normal conditions, when only a small fraction of intracellular insulin is released after glucose challenge (Kharitonenkov et al., 2013; Omar et al., 2014). However, insulin biosynthesis is an important factor for glucose-induced insulin response under diabetic conditions, characterized by a lack of adaptation of insulin biosynthesis rates to the increased insulin demand (Wente et al., 2006; Reitman, 2013). This conclusion is reinforced by our finding that FGF-21 partially rescued glucose-induced insulin secretion in rat islets that were exposed for a prolonged period of time to elevated glucose and free fatty acid levels. The loss of insulin stores has been indicated to participate in the deterioration of insulin response to glucose and, consequently, the deterioration of glucose homeostasis as a whole.

In this study, we have discovered the presence of FGF-21 in the blood of pediatric diabetes patients for the first time. In addition, FGF-21 was predicted to be the target of miR-577. We also investigated the effects of miR-577 on $\beta$-cell function and survival by targeting FGF-21.

\section{Conflicts of interest}

The authors declare no conflict of interest.

\section{REFERENCES}

Adams AC, Halstead CA, Hansen BC, Irizarry AR, et al. (2013). LY2405319, an engineered FGF21 variant, improves the metabolic status of diabetic monkeys. PLoS One 8: e65763.

Baroukh N, Ravier MA, Loder MK, Hill EV, et al. (2007). MicroRNA-124a regulates foxa2 expression and intracellular signaling in pancreatic $\beta$-cell lines. J. Biol. Chem. 282: 19575-19588.

Cong WT, Ling J, Tian HS, Ling R, et al. (2013). Proteomic study on the protective mechanism of fibroblast growth factor 21 to ischemia-reperfusion injury. Can. J. Physiol. Pharmacol. 91: 973-984.

Eswarakumar V, Lax I and Schlessinger J (2005). Cellular signaling by fibroblast growth factor receptors. Cytokine Growth Factor Rev. 16: 139-149.

Gaich G, Chien JY, Fu H, Glass LC, et al. (2013). The effects of LY2405319, an FGF21 analog, in obese human subjects with type 2 diabetes. Cell Metab. 18: 333-340.

Huang J, Ishino T, Chen G, Rolzin P, et al. (2013). Development of a novel long-acting antidiabetic FGF21 mimetic by targeted conjugation to a scaffold antibody. J. Pharmacol. Exp. Ther. 346: 270-280.

Keller DM, Clark EA and Goodman RH (2012). Regulation of microRNA-375 by cAMP in pancreatic $\beta$-cells. Mol. Endocrinol. 26: 989-999. 
Kharitonenkov A and Adams AC (2013). Inventing new medicines: The FGF21 story. Mol. Metab. 3: 221-229.

Kharitonenkov A, Shiyanova TL, Koester A, Ford AM, et al. (2005). FGF-21 as a novel metabolic regulator. J. Clin. Invest. 115: 1627-1635

Kharitonenkov A, Beals JM, Micanovic R, Strifler BA, et al. (2013). Rational design of a fibroblast growth factor 21-based clinical candidate, LY2405319. PLoS One 8: e58575.

Kim KH, Jeong YT, Kim SH, Jung HS, et al. (2013). Metformin-induced inhibition of the mitochondrial respiratory chain increases FGF21 expression via ATF4 activation. Biochem. Biophys. Res. Commun. 440: 76-81.

Nishimura T, Nakatake Y, Konishi M and Nobuyuki I (2000). Identification of a novel FGF, FGF-21, preferentially expressed in the liver. Biochim. Biophys. Acta 1492: 203-206.

Omar BA, Andersen B, Hald J, Raun K, et al. (2014). Fibroblast growth factor 21 (FGF21) and glucagon-like peptide 1 contribute to diabetes resistance in glucagon receptor-deficient mice. Diabetes 63: 101-110.

Poy MN, Hausser J, Trajkovski M, Braun M, et al. (2009). miR-375 maintains normal pancreatic alpha- and beta-cell mass. Proc. Natl. Acad. Sci. U. S. A. 106: 5813-5818.

Reitman ML (2013). FGF21 mimetic shows therapeutic promise. Cell Metab. 18: 307-309.

Schaap FG, Kremer AE, Lamers WH, Jansen PL, et al. (2013). Fibroblast growth factor 21 is induced by endoplasmic reticulum stress. Biochimie 95: 692-699.

Wang R, McGrath BC, Kopp RF, Roe MW, et al. (2013). Insulin secretion and Ca2+ dynamics in $\beta$-cells are regulated by PERK elF2 $\alpha$ kinase in concert with calcineurin. J. Biol. Chem. 288: 33824-33836.

Wente W, Efanov AM, Brenner M, Kharitonenkov A, et al. (2006). Fibroblast growth factor-21 improves pancreatic beta-cell function and survival by activation of extracellular signal-regulated kinase $1 / 2$ and Akt signaling pathways. Diabetes 55 : 2470-2478.

Zheng X, Zheng X, Wang X, Ma Z, et al. (2012). Acute hypoxia induces apoptosis of pancreatic $\beta$-cell by activation of the unfolded protein response and upregulation of CHOP. Cell Death Dis. 3: e322. 